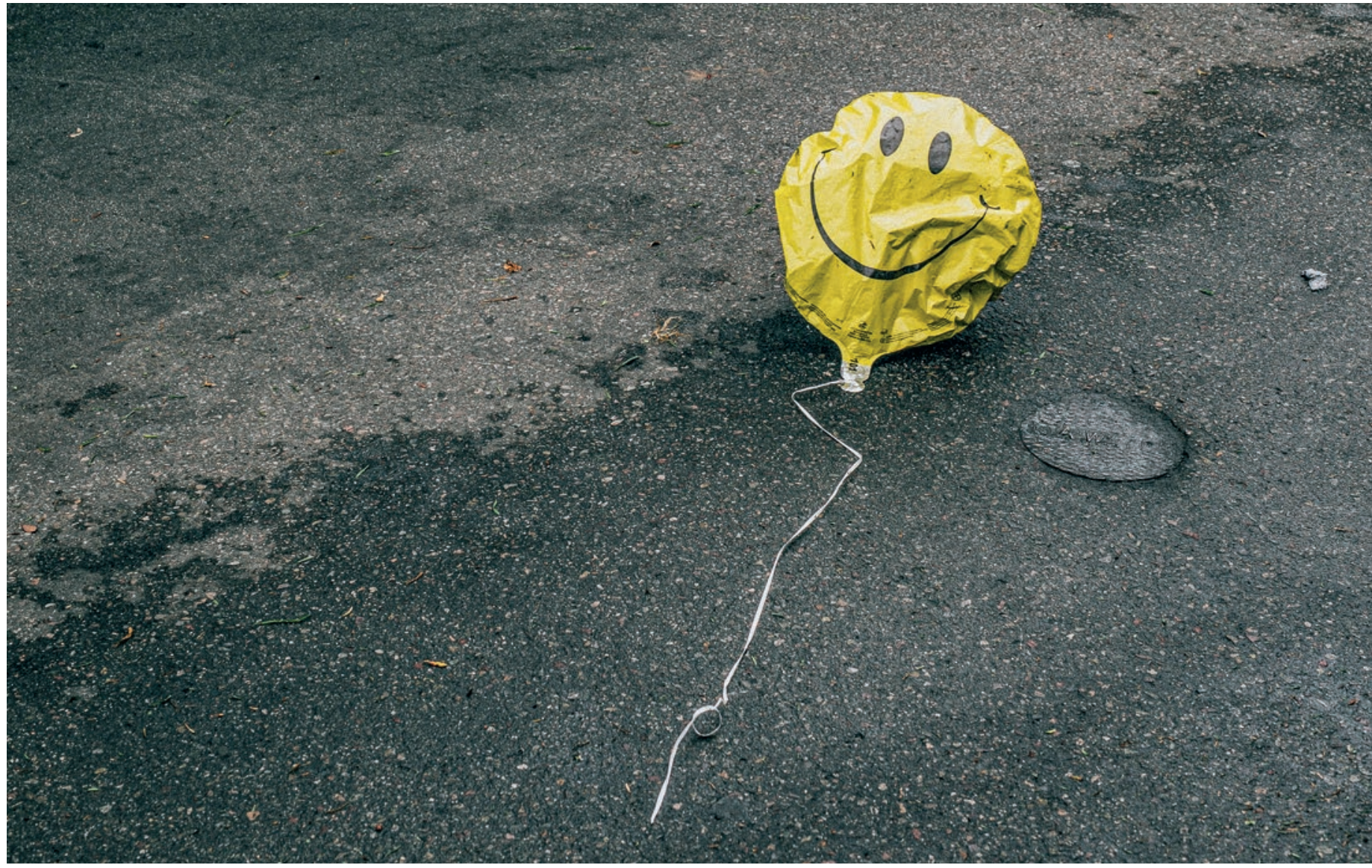

\title{
Schlechte Stimmung, aber noch immer viel Vertrauen in die Politik
}

\section{Redaktion Schweizerische Ärztezeitung}

11,3\% der Bevölkerung waren in der ersten Jahreshälfte 2021 aufgrund der Pandemie mit Einkommenseinbussen konfrontiert, teilt das Bundesamt für Statistik mit. Betroffen waren vor allem Personen, die schon vor der Krise benachteiligt waren. Und insbesondere junge Menschen berichten von einem negativen Einfluss der Covid-19-Pandemie auf ihre Stimmungslage.

20\% der Bevölkerung lebten in der ersten Hälfte des Jahres 2021 in einem Haushalt, dessen gesamtes Einkommen nach eigener Einschätzung in den letzten 12 Monaten gesunken ist. Mehr als die Hälfte davon (11,3\%) gab die Covid-19-Pandemie als Begründung hierfür an, wie eine aktuelle Erhebung des Bundesamts für Statistik zeigt. [1] Die Pandemie führte besonders häufig zu Einbussen bei Personen, die im Bereich Gastgewerbe und Beherbergung tätig sind
(35,5\%), sowie bei Personen mit niedrigen selbsteingeschätzten Einkommen (19,5\%) und auch bei ausländischen Personen (16,7\%). Beschäftigte in der öffentlichen Verwaltung oder im Bereich Erziehung und Unterreicht waren dagegen weniger betroffen $(4,2$ bzw. 8,2\%).

Dennoch nahm laut dem Bundesamt für Statistik der Anteil Personen, die leicht oder sehr leicht über die Runden kommen, zwischen 2019 und 2021 von 48,4 auf 


\section{Zufriedenheit in verschiedenen Lebensbereichen}

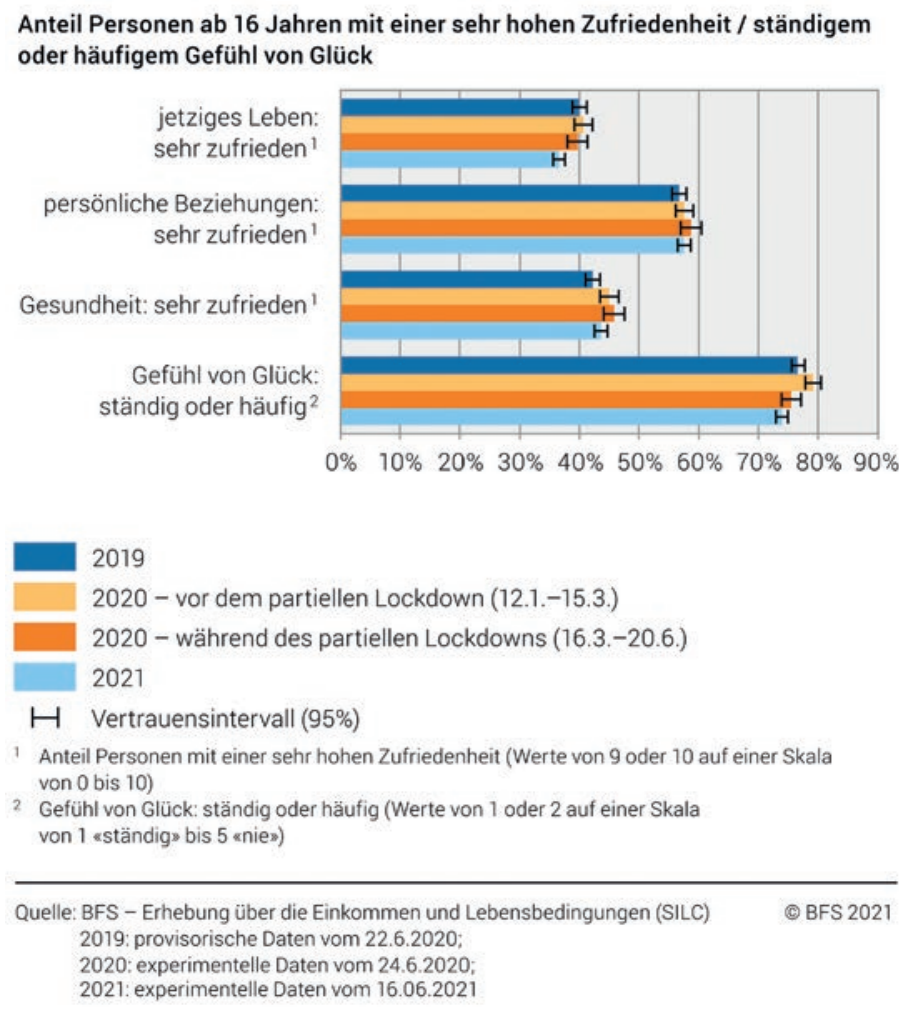

Anteil Personen ab 16 Jahren mit einer sehr hohen Zufriedenheit / ständigem oder häufigem Gefühl von Glück

2019

2020 - vor dem partiellen Lockdown (12.1.-15.3.)

2020 - während des partiellen Lockdowns (16.3.-20.6.)

2021

$\mapsto$ Vertrauensintervall (95\%)

Anteil Personen mit einer sehr hohen Zufriedenheit (Werte von 9 oder 10 auf einer Skala von 0 bis 10)

2 Gefühl von Glück: ständig oder häufig (Werte von 1 oder 2 auf einer Skala von 1 «ständig» bis 5 «nies)

Quelle: BFS - Erhebung über die Einkommen und Lebensbedingungen (SILC)

() BFS 2021

2019: provisorische Daten vom 22.6.2020:

2020: experimentelle Daten vom 24.6.2020

2021: experimentelle Daten vom 16.06.2021

\section{Subjektive Einschätzung der finanziellen Situation des Haushalts}

Anteil Personen, die in einem Haushalt leben, für den es leicht oder sehr leicht ist, finanziell über die Runden zu kommen

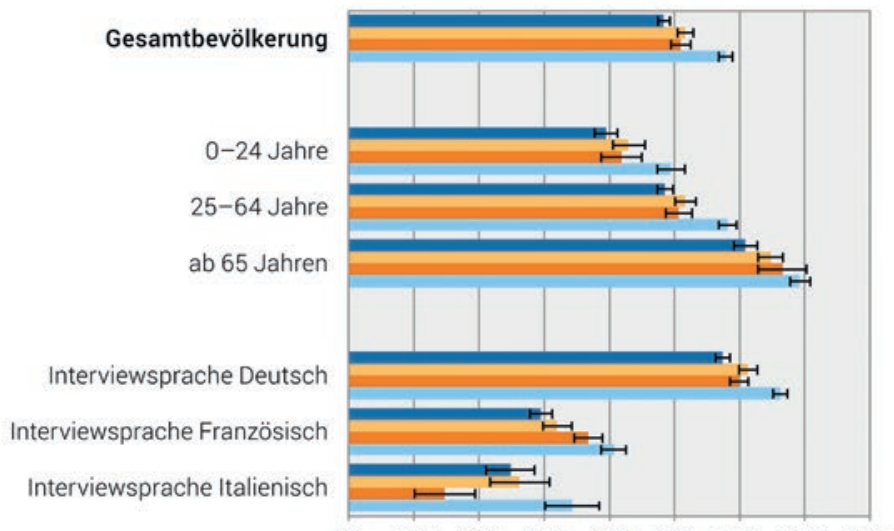

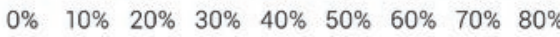

2019

2020 - vor dem partiellen Lockdown (12.1.-15.3.)

2020 - während des partiellen Lockdowns (16.3.-20.6.)

2021

$\mapsto$ Vertrauensintervall (95\%)

Quelle: BFS - Erhebung über die Einkommen und Lebensbedingungen (SILC) 2019: provisorische Daten vom 22.6 .2020

2020: experimentelle Daten vom 24.6.2020;

2021: experimentelle Daten vom 16.06.2021

Laut dem Bundesamt für Statistik ist die allgemeine Zufriedenheit zurückgegangen. Gleichzeitig kommen mehr Menschen finanziell gut über die Runden.

$57,9 \% \mathrm{zu}$, was sich neben einem allgemeinen Rückgang des Konsums unter anderem auch mit einem häufigeren Verzicht auf Freizeitaktivitäten (zum Beispiel Restaurantbesuche, Sport oder kulturelle Aktivitäten) in dieser Zeitspanne erklären lässt.

\section{Negative Stimmungslage bei den Jungen}

Die Gesundheitskrise hat auch negative Folgen auf die psychische Gesundheit der Bevölkerung in der Schweiz: 40,2\% gaben in der ersten Hälfte des Jahres 2021 an, dass sich die Covid-19-Pandemie negativ auf ihre Stimmungslage ausgewirkt hat.

\section{0,2\% der Befragten sagten, dass sich} die Covid-19-Pandemie negativ auf ihre Stimmung ausgewirkt hat.

Der Anteil war besonders hoch bei Personen zwischen 16 und 24 Jahren (55,1\%), Personen mit einer tertiären Ausbildung (44,8\%) und den Personen mit einem höheren selbsteingeschätzten Einkommen (45,1\%). Dagegen hatte die Gesundheitskrise weniger negative Auswirkungen auf die Stimmungslage der Personen in dünn besiedelten Gebieten (36,4\%) und Personen über 65 Jahre (26,0\%).

\section{Unterschiede beim Thema Homeoffice}

Fast 50\% der erwerbstätigen Bevölkerung hatten seit Beginn der Pandemie immer oder zumindest zeitweise die Möglichkeit, zu Hause zu arbeiten. Aber auch hier zeigen sich grosse Unterschiede zwischen den Bevölkerungsgruppen.

Während 67,7\% der Personen mit Tertiärabschluss und 72,3\% der Personen mit höherem selbsteingeschätzten Einkommen deutlich häufiger immer oder zeitweise im Homeoffice arbeiten konnten, war dies nur bei 39,9\% der ausländischen Staatsbürgerinnen und -bürger, bei 31,7\% der Personen mit einem niedrigen selbsteingeschätzten Einkommen und bei 16,6\% der Personen ohne nachobligatorische Ausbildung der Fall.

\section{Weniger Sorgen um den Arbeitsplatz}

Zu Beginn der Gesundheitskrise wurden aber auch Sorgen bezüglich der künftigen finanziellen Situation geäussert, insbesondere eine deutlich geringere Arbeits- 
platzsicherheit. Nach einem starken Rückgang der subjektiven Einschätzung der Arbeitsplatzsicherheit während des partiellen Lockdowns im Jahr 2020 ist diese 2021 wieder gestiegen: Der Anteil der erwerbstätigen Bevölkerung, die das Risiko, den Arbeitsplatz zu verlieren, als sehr gering einschätzt, stieg von $53,5 \%$ während des partiellen Lockdowns auf 60,5\% im Jahr 2021 an, blieb jedoch signifikant unter dem Niveau von 2019 (64,6\%).

\section{Seit Beginn der Pandemie nahm der Anteil Personen, die sich häufig glücklich fühlen, signifikant $a b$.}

Die subjektive Arbeitsplatzsicherheit erlangte bei den Personen mit Schweizer Nationalität, Tertiärabschluss und einem hohen selbsteingeschätzten Einkommen beinahe wieder das Niveau von vor der Gesundheitskrise. Dagegen wurde dieses Niveau bei den Personen mit ausländischer Nationalität, den französischsprachigen Personen und den Personen mit einem niedrigen selbsteingeschätzten Einkommen laut dem Bundesamt für Statistik deutlich nicht wieder erreicht.

\section{Menschen sind nicht immer glücklich}

Seit Beginn der Gesundheitskrise nahm der Anteil Personen, die sich ständig oder häufig glücklich fühlen, si- gnifikant ab und betrug in der ersten Jahreshälfte 2021 noch $73,9 \%$ (gegenüber 79,2\% vor dem partiellen Lockdown 2020).

In der gleichen Zeit sank der Anteil Personen mit einer sehr hohen Zufriedenheit mit dem jetzigen Leben von 40,7 auf 36,6\%. Dagegen veränderte sich die allgemein hohe Zufriedenheit mit den persönlichen Beziehungen und dem wahrgenommenen Gesundheitszustand der Bevölkerung in der Schweiz kaum.

\section{Politik geniesst Vertrauen}

Das Vertrauen der Bevölkerung in das politische System ist in der Anfangszeit der Covid-19-Pandemie deutlich gestiegen. Der Anteil Personen mit hohem oder sehr hohem Vertrauen in das politische System in der Schweiz stieg von $47,5 \%$ vor dem partiellen Lockdown auf 54,0\% während des partiellen Lockdowns an.

Dieser Vertrauensgewinn in das politische System flachte im ersten Halbjahr 2021 zwar wieder leicht ab, blieb aber auf einem höheren Niveau als noch vor dem Beginn der Gesundheitskrise.

\section{Literatur}

1 Experimentelle Statistiken: Covid-19 und Lebensbedingungen in der Schweiz 2021 (SILC) | Bundesamt für Statistik: www.bfs.admin. ch/news/de/2021-0627

Bildnachweis Einstiegsbild

Nathan Dumlao / Unsplash 